\title{
INTERAÇÃO ENTRE CULTIVARES, ESTIRPES COMERCIAIS DE RHIZOBIUM MELILOTIE FUNGICIDAS NO INCREMENTO DA PRODUÇÃO DE ALFAFA ${ }^{1}$
}

\author{
PATRÍCIA PERONDI ANCHÃO OLIVEIRA², SIU MUI TSAI ${ }^{3}$, MOACYR CORSI $^{4}$ e MARÍA DEL PILAR DÍAZ ${ }^{5}$
}

RESUMO - A cultura da alfafa (Medicago sativa) é importante para a produção animal, por sua alta qualidade nutritiva, alto potencial de produção e sucesso na fixação de nitrogênio, através da simbiose com o Rhizobium meliloti, dispensando o uso de adubação nitrogenada. Neste trabalho objetivou-se selecionar estirpes comerciais de $R$. meliloti (SEMIA-116, SEMIA-134 e SEMIA-135) em relação às cultivares de alfafa Flórida 77, Pioneer 5929, CUF 101 e Crioula, e avaliar os fungicidas Iprodione e Thiram em tratamento de semente e solo na cultivar Crioula. Não houve interação entre as estirpes de R. meliloti e as cultivares de alfafa quanto à produção de matéria seca, e as plantas infestadas com a SEMIA-116 apresentaram maior número de nódulos. Houve interações entre os diferentes tipos e doses de fungicidas e as estirpes de $R$. meliloti testadas. A estirpe SEMIA-116 apresentou superioridade, representada pela maior produção de biomassa e número de nódulos. Pelos resultados obtidos, não se recomenda a inoculação da estirpe SEMIA-135, quando em associação com o fungicida Iprodione, em solo ou semente.

Termos para indexação: Medicago sativa, nitrogênio, nodulação, tratamento de sementes.

\section{INTERACTIONS OF CULTIVARS, COMMERCIAL STRAINS OF RHIZOBIUM MELILOTI AND FUNGICIDES ON THE PRODUCTION OF ALFALFA}

\begin{abstract}
The cultivation of alfalfa (Medicago sativa) is not only important because of its nutrient, content, but also for its high productivity and its ability to fix nitrogen through its association with Rhizobium meliloti. To better understand this symbiotic association, the interaction between $R$. meliloti strains SEMIA-116, SEMIA-134 and SEMIA-135 with different cultivars of alfalfa Florida 77, Pioneer 5929, CUF 101 and Crioula was evaluated. In addition to the above treatments, the fungicides Iprodione and Thiram were used with the cultivar Crioula to study their effects on the alfalfa/Rhizobium interaction. Plants inoculated with SEMIA-116 had a greater number of nodules. However, no significant difference in the production of plant dry matter was observed between the various Rhizobium strains and the alfalfa cultivars tested. In the presence of the tested fungicides, the Crioula/SEMIA-116 association showed superior biomass production and number of nodules. Results obtained in this study suggest that SEMIA-135 should not be used as an inoculum for alfalfa when Iprodione is used either as a soil or seed treatment.
\end{abstract}

Index terms: Medicago sativa, nitrogen, nodulation, seed treatment.

\footnotetext{
${ }^{1}$ Aceito para publicação em 20 de janeiro de 1998. Trabalho financiado pela FAPESP.

${ }^{2}$ Eng. Agr., M.Sc., Dep. Zootecnia, ESALQ-USP, Caixa Postal 09, CEP 13418-900 Piracicaba, SP. E-mail: ppaolive@pira.cena.usp.br

${ }^{3}$ Enga ${ }^{\mathrm{a}}$ Agr ${ }^{\mathrm{a}}, \mathrm{Dr}^{\mathrm{a}}$, Lab. de Microbiologia, CENA/USP, Caixa Postal 96, CEP 13400-970 Piracicaba, SP. E-mail: tsai@cena.usp.br

${ }^{4}$ Eng. Agr., Dr., Prof. Titular, Dep. Zootecnia, ESALQ-USP.

${ }^{5}$ Matemática, Dra , Facultad de Ciencias Agropecuarias de la Universidad Nacional de Córdoba, Caja Postal 509, CP 5016, Córdoba, Argentina. E-mail: pdiaz@agro.uncor.edu
}

\section{INTRODUÇÃO}

O cultivo da alfafa (Medicago sativa) é bastante restrito no Brasil. Apesar de todo o País apresentar condições de boa produção, a maior parte desse cultivo está concentrada na Região Sul. O uso da alfafa como fonte de alimento deveria ser mais difundido nas outras regiões brasileiras, por tratar-se de uma forrageira muito importante, tendo em vista sua capacidade de fixação biológica de $\mathrm{N}$, que significa o fornecimento de alimentos com maiores teores 
protéicos para os animais, sem a necessidade do uso de fertilizantes nitrogenados. A seleção de estirpes de rizóbio adequadas para a eficiência do processo de fixação de $\mathrm{N}$ é fator imprescindível para garantir altas produtividades, persistência do alfafal e qualidade da forragem colhida.

É reconhecida a existência da interação entre estirpes de Rhizobium meliloti e cultivares da alfafa com diferentes resultados quanto à eficiência da fixação de N atmosférico (Vance et al., 1988; ArreseIgor et al., 1989; Twary \& Heichel, 1991). No Brasil, Kolling et al. (1983) testaram as estirpes SEMIA-108 (inoculante Nitragin, USA); SEMIA-115 (USDA 1082, USA); SEMIA-116(USDA 1088, USA); SEMIA-113 (SU 47, Austrália); SEMIA-134 (isolamento RS, Brasil) e SEMIA-135 (isolamento RS, Brasil) em relação à cultivar Crioula, e observaram que as estirpes SEMIA-115, SEMIA-116, SEMIA-113, SEMIA-134 e SEMIA-135 revelaram alta eficiência no rendimento de matéria seca, enquanto a estirpe SEMIA-108 foi a menos eficiente no primeiro ano. Nos anos subseqüentes, a SEMIA-134 apresentou menor eficiência que as demais estirpes de alta eficiência no primeiro ano.

Com o objetivo de garantir o estabelecimento da cultura da alfafa, os fungicidas Thiram e Iprodione têm sido utilizados no tratamento de sementes e de solo. O fungicida Thiram é recomendado em tratamento de semente no Sul do País (Keplin \& Santos, 1991), e o Iprodione foi utilizado em tratamento de sementes e de solo para o controle de damping-off causado por Rhizoctonia solani (Compêndio..., 1993). O uso de fungicidas no tratamento de sementes e de solo coloca esses produtos em contato com o inoculante, fato que pode ser prejudicial à sobrevivência do inóculo, pelos possíveis efeitos nocivos do princípio ativo do produto.

O efeito biológico do tratamento de sementes de leguminosas dependerá da dose de fungicida aplicada, da estirpe de Rhizobium utilizada, de fatores climáticos, e de outros microrganismos existentes na rizosfera. Tal efeito pode ser depressivo, não influenciar ou incrementar os fatores produtivos de uma cultura (De-Polli et al., 1986; Isoi \& Yoshida, 1988).
Tu (1981) avaliou cinco fungicidas (Benomyl, Captan, Maneb, Thiram, Zineb) em relação à cultura da alfafa, e observou que o Thiram foi tóxico sobre o crescimento da bactéria em disco de papelfiltro. Com relação ao desenvolvimento e crescimento das plantas em vasos, observou que elevados níveis de fungicidas suprimiram a nodulação quando comparadas com plantas não tratadas ou tratadas com dosagens baixas. O fungicida Thiram provocou inibição na fixação de $\mathrm{N}$, causando redução no crescimento da parte aérea, nas dosagens de $3 \mathrm{mg} / \mathrm{g}$ de semente, e no crescimento de nódulos, nas dosagens de 2,5 e $3 \mathrm{mg} / \mathrm{g}$ de semente. Por sua vez o fungicida estimulou o crescimento da parte aérea e da raiz quando aplicado de 0,2 a $0,5 \mathrm{mg} / \mathrm{g}$ de semente. $\mathrm{O}$ autor considera que os resultados obtidos em laboratório, com relação ao efeito de fungicidas sobre o Rhizobium, devem ser vistos com cautela quando se pretende transferi-los para o campo.

Odeyemi \& Alexander (1977) ressaltaram a importância da estirpe de R. meliloti e sua interação com o uso de fungicidas, por meio do trabalho de isolamento de estirpes resistentes ao Thiram, a partir de cultura de estirpe sensível. A técnica utilizada foi a obtenção da resistência pelo cultivo em meios contendo quantidades crescentes de fungicidas. As plantas cultivadas com a estirpe resistente, na presença do Thiram ( $5,0 \mathrm{mg} / \mathrm{g}$ de semente), cresceram tão bem quanto as que não receberam fungicida no tratamento de semente. As plantas oriundas de sementes tratadas com Thiram e infectadas com Rhizobium nãoresistente cresceram atrofiadas, cloróticas e com pequena ou ausente fixação de N.

Måtensson (1992) estudou o Rhizobium meliloti com o objetivo de testar os efeitos de alguns fungicidas (Fenpropimorph, Mancozeb e Benomyl) e metais pesados sobre o crescimento dessa bactéria e sua simbiose com a alfafa. O autor utilizou duas estirpes de Rhizobium meliloti (a RME, isolada de um solo com manejo ecológico e a RMF, isolada de um solo exposto ao uso de agroquímicos) e vários métodos para promover a avaliação das estirpes. Neste caso, o autor não encontrou diferenças nas respostas das diferentes estirpes utilizadas quanto à presença dos fungicidas testados.

Desta forma, observa-se que as conseqüências do uso de fungicidas no tratamento de sementes e 
de solo na cultura da alfafa, sobre a simbiose com o Rhizobium meliloti, parecem estar extremamente ligadas à estirpe utilizada.

O objetivo deste trabalho foi selecionar estirpes comerciais de Rhizobium meliloti utilizadas no Brasil em diferentes cultivares de alfafa e avaliar a interação dessas estirpes com os fungicidas Thiram e Iprodione em tratamento de sementes e de solo na cultivar Crioula.

\section{MATERIAL E MÉTODOS}

O presente estudo foi realizado em Piracicaba, SP, com dois experimentos, em casa de vegetação. Um deles foi conduzido no Departamento de Horticultura, ESALQ/ USP, em vasos com solo, para avaliar a eficiência das três estirpes comerciais de Rhizobium meliloti SEMIA-116, SEMIA-134 e SEMIA-135 associadas às quatro cultivares de alfafa, Flórida 77, CUF 101, Crioula e Pioneer 5929, sendo a Crioula uma cultivar brasileira e as demais norteamericanas; e outro no Centro de Energia Nuclear na Agricultura/USP, em vasos Leonard, para avaliar o uso de tratamentos de solo e sementes com os fungicidas Iprodione e Thiram, associados à inoculação das estirpes testadas em relação à cultivar Crioula.

\section{Estirpes de Rhizobium meliloti $\mathrm{x}$ cultivares de alfafa}

O delineamento experimental utilizado foi o de blocos inteiramente casualizados, com cinco repetições, em arranjo fatorial de três estirpes de Rhizobium meliloti e quatro cultivares de alfafa. Como testemunha foram utilizados um tratamento sem inoculação e outro sem inoculação acrescido de $50 \mathrm{~kg} / \mathrm{ha} \mathrm{de} \mathrm{N}$.

$\mathrm{O}$ experimento foi conduzido em vasos com capacidade para três litros de solo (Latossolo Vermelho-Amarelo) autoclavado. Os resultados da análise química do solo foram: $\mathrm{pH} \mathrm{CaCl} 2=4,2 ; \mathrm{MO}=14 \mathrm{~g} / \mathrm{dm}^{3} ; \mathrm{P}$ (resina) $=3 \mathrm{mg} / \mathrm{dm}^{3}$; $\mathrm{K}=0,3, \mathrm{Ca}=14,0, \mathrm{Mg}=2,0$ e $\mathrm{H}+\mathrm{Al}=64,0 \mathrm{mmol}_{\mathrm{c}} \mathrm{dm}^{3}$. Esse solo recebeu $6,9 \mathrm{t} / \mathrm{ha}$ de calcário dolomítico $\mathrm{PRNT}=70 \% \mathrm{e}$ ficou incubado durante um mês antes do plantio. Por ocasião do plantio, foram aplicados $120 \mathrm{~kg} / \mathrm{ha}$ de $\mathrm{P}_{2} \mathrm{O}_{5}$ e 200 $\mathrm{kg} /$ ha de $\mathrm{K}_{2} \mathrm{O}$ mais solução de micronutrientes. Nos vasos do tratamento com N, foi aplicado o equivalente a $50 \mathrm{~kg} / \mathrm{ha}$ de $\mathrm{N}$ na forma de uréia, assim que as plantas começaram a emitir a segunda haste.

Em cada vaso foram colocadas cinco sementes, infectadas com a estirpe respectiva ao seu tratamento. O inóculo foi previamente crescido em meio YM, sendo poste- riormente veiculado em turfa irradiada, no Laboratório de Microbiologia do CENA/USP, e utilizado na proporção de $6 \mathrm{~g} / \mathrm{kg}$ de semente. Todos os lotes foram tratados com quantidades iguais de carbonato de cálcio e água. Foi realizado um desbaste, permanecendo uma planta por vaso. A umidade dos vasos foi mantida próxima à capacidade de campo.

Os parâmetros avaliados foram biomassa seca da parte aérea, biomassa seca da raiz, número de nódulos e porcentagem de $\mathrm{N}$ da parte aérea. Os dados foram submetidos à análise de variância, e as médias, comparadas pelos testes de Tukey ou de REGWQ, 5\% de probabilidade.

\section{Estirpes de Rhizobium meliloti x fungicidas}

O delineamento experimental foi o de blocos inteiramente casualizados, com cinco repetições, em arranjo fatorial de $4 \times 3$ tratamentos. Foram avaliadas quatro formas de aplicação de fungicida: Thiram, 4,2 g p.a. $/ \mathrm{kg}$ de semente, na forma de Rhodiauram em tratamento de semente; Iprodione, 1 g p.a. $/ \mathrm{kg}$ de semente, na forma de Rovral, em tratamento de semente; Iprodione, 37,5 g p.a./kg de semente, na forma de Rovral aplicado ao solo; e três estirpes de Rhizobium meliloti: SEMIA-116, SEMIA-134 e SEMIA-135. O controle consistiu de um tratamento sem fungicida.

$\mathrm{O}$ tratamento de sementes foi realizado em lotes, onde os inóculos e os fungicidas foram aplicados simultaneamente. A inoculação foi realizada conforme descrição anterior. A aplicação do fungicida Rovral ao solo foi realizada por meio de irrigação da superfície dos vasos Leonard, com uma pipeta, sete dias após a emergência das plantas. $\mathrm{Na}$ semeadura foram colocadas cinco sementes por vaso de alfafa, cv. Crioula, sendo realizado um desbaste deixando-se duas plantas por vaso.

Foi utilizada solução nutritiva (Sarruge, 1975) exceto $\mathrm{N}(500 \mathrm{~mL})$ no vaso Leonard, com capacidade de $900 \mathrm{~mL}$, na diluição 1:5 em água, nos primeiros 15 dias após plantio, e depois, diluição 1:3, até a colheita. As reposições foram semanais.

Foram avaliados os parâmetros: altura das plantas aos 60 dias após a emergência (DAE), medida a partir do nível da inserção das raízes na época da colheita; biomassa seca da parte aérea; porcentagem de $\mathrm{N}$ na parte aérea; biomassa seca das raízes; número de nódulos e biomassa seca dos nódulos. Para secagem das amostras utilizouse estufa de circulação de ar forçada, a $65^{\circ} \mathrm{C}$, durante 48 horas.

Os dados do experimento foram submetidos à análise de variância e as médias comparadas pelos testes de Tukey 
e REGWQ (Ryan-Gabriel-Einot-Welsch) recomendado por SAS (1989), a 5\% de probabilidade.

\section{RESULTADOS E DISCUSSÃO}

\section{Estirpes de Rhizobium meliloti $\mathrm{x}$ cultivares de alfafa}

Não houve interação entre as estirpes de $R$. meliloti e as cultivares de alfafa testadas quanto aos parâmetros biomassa seca da parte aérea, número de nódulos e porcentagem de N. Entretanto, houve diferenças entre as estirpes de $R$. meliloti e os tratamentos controle e com adição de N. A porcentagem de $\mathrm{N}$ foi superior nos tratamentos com inoculação da SEMIA-134 e $50 \mathrm{~kg} /$ ha de N, resultando em maior quantidade de $\mathrm{N}$ colocada no sistema. Em relação à matéria seca da parte aérea, não houve diferença entre as estirpes testadas e o tratamento com $50 \mathrm{~kg} / \mathrm{ha}$ de $\mathrm{N}$, e o controle apresentou resultado inferior a inoculação com a estirpe SEMIA-135. O tratamento inoculado com a SEMIA 116 apresentou número significativamente maior de nódulos que os outros tratamentos, sendo que nos tratamentos sem inoculação não se detectou a presença de nódulos (Tabela 1).

Os valores obtidos coincidem com os observados por Kolling et al. (1983), que utilizaram as mesmas estirpes de Rhizobium e a cultivar Crioula. $\mathrm{O}$ fato de não ter sido encontrada interação entre as estirpes e as cultivares neste trabalho não significa que o mesmo comportamento seja esperado, em outras condições, em relação a outras estirpes e cultivares, conforme sugerido por Vance et al. (1988).

\section{Estirpes de Rhizobium meliloti x uso de fungicidas}

Para avaliação dos efeitos dos fungicidas sobre a eficiência simbiótica com as estirpes de Rhizobium, foram medidos biomassa seca da raiz, número de nódulos e biomassa seca dos nódulos (Tabela 2). Quanto à biomassa seca da raiz, dois tratamentos se destacaram: a estirpe SEMIA-116 combinada com fungicida Thiram, que apresentou a produção mais alta de raízes (438 mg) e a SEMIA-135, na presença de Iprodione em superfície, com o menor resultado (76 mg). Em relação à biomassa seca dos nódulos, a inoculação da SEMIA-135 e o uso de Iprodione em superfície proporcionaram o resultado mais baixo $(1,20 \mathrm{mg})$, e o melhor resultado foi apresentado no tratamento SEMIA-116 combinada com o fungicida Iprodione em superfície. Observa-se que a SEMIA-116 não diferiu em presença de qualquer tipo de fungicida. A estirpe SEMIA-134 apresentou-se diferente da SEMIA-116 apenas quando associada com o fungicida Thiram. O uso do fungicida Iprodione em superfície trouxe o pior resultado quando associado à estirpe SEMIA-135. Quando utilizadas na ausência de fungicidas, as três estirpes não

TABELA 1. Valores médios da biomassa seca da parte aérea, porcentagem de nitrogênio e número de nódulos em quatro cultivares de alfafa (Crioula, Flórida 77, CUF 101, Pioneer 5929) ${ }^{1}$.

\begin{tabular}{|c|c|c|c|}
\hline Tratamento & Parte aérea $(\mathrm{mg})^{2}$ & Nitrogênio $(\%)^{2}$ & Nódulos $\left(\mathrm{n}^{\mathrm{o}}\right)^{3}$ \\
\hline Testemunha & $1,20 \mathrm{~b}$ & $2,49 b$ & - \\
\hline Adubação com $50 \mathrm{~kg} / \mathrm{ha}$ de N & $1,52 \mathrm{ab}$ & $3,48 \mathrm{a}$ & - \\
\hline Inoculação $R$. meliloti SEMIA-116 & $1,60 \mathrm{ab}$ & $2,19 b$ & $54,95 \mathrm{a}$ \\
\hline Inoculação $R$. meliloti SEMIA-134 & $1,64 \mathrm{ab}$ & $3,18 \mathrm{a}$ & $26,25 b$ \\
\hline Inoculação $R$. meliloti SEMIA-135 & $1,94 \mathrm{a}$ & $2,34 b$ & $25,15 b$ \\
\hline Média geral & 1,58 & 2,75 & 35,45 \\
\hline $\mathrm{CV}(\%)$ & 35,94 & 19,10 & 43,50 \\
\hline
\end{tabular}

${ }^{1}$ Médias seguidas pela mesma letra não diferem entre si a $5 \%$ de probabilidade.

2 Teste de REGWQ para diferenciação das médias (SAS, 1989).

${ }^{3}$ Teste de Tukey para diferenciação das médias (SAS, 1989). 
diferiram entre si quanto à capacidade de formação de nódulos.

A fixação de $\mathrm{N}$ é influenciada pela dose de fungicida empregada, pois dependendo de sua concentração e da reação da estirpe utilizada ocorre interação quanto ao N (Odeyemi \& Alexander, 1977; Tu, 1981; Rennie et al., 1985; Isoi \& Yoshida, 1988). $\mathrm{Tu}$ (1981) encontrou inibição do processo de infecção, nodulação e fixação de $\mathrm{N}$ quando trabalhou com doses de 2,5 a 3,0 $\mathrm{mg}$ de Thiram/g de semente, e estímulo do processo quando trabalhou com doses entre 0,2 e 0,5 mg. Entretanto, no presente trabalho, a SEMIA-116 apresentou tendência de melhor resistência ao uso dos fungicidas testados, principalmente Thiram. Odeyemi \& Alexander (1977) também encontraram estirpes de Rhizobium meliloti resistentes ao uso de Thiram em dose semelhante à deste trabalho, ou seja, de $5 \mathrm{mg}$ de Thiram/g de semente.

A biomassa seca da parte aérea e a altura das plantas podem ser obsevadas na Tabela 3. A biomassa seca da parte aérea apresentou comportamento dependente das estirpes e dos fungicidas utilizados.

TABELA 2. Valores médios da biomassa seca da raiz e dos nódulos e número de nódulos de alfafa cv. Crioula submetidas à inoculação de três estirpes de rizóbio e a tratamentos com dois fungicidas ${ }^{1}$.

\begin{tabular}{|c|c|c|c|c|}
\hline $\begin{array}{l}\text { Estirpe de } \\
\text { rizóbio }\end{array}$ & Fungicida $^{2}$ & $\begin{array}{l}\mathrm{Raiz}^{3} \\
(\mathrm{mg})\end{array}$ & $\begin{array}{c}\text { Nódulo }^{4} \\
\text { (mg) }\end{array}$ & $\begin{array}{c}\text { Nódulo } \\
\left(\mathrm{n}^{\mathrm{o}}\right)\end{array}$ \\
\hline SEMIA-116 & Controle & $172 \mathrm{bc}$ & $22,5 \mathrm{abc}$ & $89,6 \mathrm{abc}$ \\
\hline SEMIA-116 & Thiram s & $438 \mathrm{a}$ & $32,9 \mathrm{abc}$ & $117,8 \mathrm{ab}$ \\
\hline SEMIA-116 & Iprodione s & $190 \mathrm{abc}$ & $29,9 \mathrm{abc}$ & $102,8 \mathrm{ab}$ \\
\hline SEMIA-116 & Iprodione ss & $366 a b$ & $42,5 \mathrm{a}$ & $168,6 \mathrm{a}$ \\
\hline SEMIA-134 & Controle & $168 \mathrm{bc}$ & $17,8 \mathrm{abc}$ & 48,0abcd \\
\hline SEMIA-134 & Thiram s & $134 b c$ & $8,6 \mathrm{bc}$ & $10,4 \mathrm{de}$ \\
\hline SEMIA-134 & Iprodione s & $236 \mathrm{abc}$ & $35,5 \mathrm{abc}$ & $71,8 \mathrm{abc}$ \\
\hline SEMIA-134 & Iprodione ss & $264 \mathrm{abc}$ & $29,7 \mathrm{abc}$ & $58,6 \mathrm{abcd}$ \\
\hline SEMIA-135 & Controle & $206 a b c$ & $25,5 \mathrm{abc}$ & $54,6 \mathrm{abcd}$ \\
\hline SEMIA-135 & Thiram s & $130 \mathrm{bc}$ & $11,1 \mathrm{abc}$ & $20,6 \mathrm{cde}$ \\
\hline SEMIA-135 & Iprodine s & $174 \mathrm{bc}$ & $13,3 \mathrm{abc}$ & $17,2 \mathrm{bcde}$ \\
\hline SEMIA-135 & Iprodione ss & $76 \mathrm{c}$ & $1,2 \mathrm{c}$ & $0,2 \mathrm{e}$ \\
\hline Média geral & & 216 & 22,6 & 63,3 \\
\hline CV $(\%)$ & & 56 & 66 & 47 \\
\hline
\end{tabular}

${ }^{1}$ Médias seguidas pela mesma letra não diferem entre si a $5 \%$ de probabilidade (Tukey e REGWQ).

${ }^{2} \mathrm{~s}$ : fungicida aplicado na semente; ss: fungicida aplicado na semente e na superfície do solo.

${ }^{3}$ Teste de Tukey para diferenciação das médias (SAS, 1989).

${ }^{4}$ Teste de REGWQ para diferenciação das médias (SAS, 1989).
Os resultados indicaram que, quanto aos tratamentos-controle e Iprodione aplicado nas sementes, não houve diferença entre as estirpes utilizadas. Quando usou-se o fungicida Thiram, a estirpe SEMIA-116 foi superior à SEMIA-135, mas ambas não diferiram da SEMIA-134. Em relação ao Iprodione aplicado na semente e na superfície do solo, as estirpes SEMIA-116 e SEMIA-134 foram superiores a SEMIA-135. A altura das plantas também apresentou interação significativa entre os fatores estirpe $\mathrm{x}$ fungicida. Quanto ao Iprodione aplicado na semente e na superfície do solo, também houve superioridade significativa das estirpes SEMIA-116 e SEMIA-134 em relação à estirpe SEMIA-135.

A análise de variância não detectou interação significativa entre as estirpes e os fungicidas quanto à porcentagem de $\mathrm{N}$ e $\mathrm{N}$ total. Entretanto, houve diferenças significativas entre as estirpes utilizadas $(\mathrm{P} \leq 0,05)$. Os resultados da aplicação do teste REGWQ para detectar a diferenciação entre as estirpes podem ser observados na Tabela 4. Houve superioridade dos tratamentos que utilizaram a inoculação da SEMIA-116 em relação à

TABELA 3. Efeito da inoculação e aplicação de fungicidas na biomassa seca da parte aérea e na altura das plantas em alfafa $\mathrm{cv}$. Crioula ${ }^{1}$.

\begin{tabular}{|c|c|c|c|}
\hline $\begin{array}{l}\text { Estirpe de } \\
\text { rizóbio }\end{array}$ & Fungicida $^{2}$ & $\begin{array}{c}\text { Parte aérea }{ }^{3} \\
(\mathrm{mg})\end{array}$ & $\begin{array}{c}\text { Altura }^{4} \\
(\mathrm{~cm})\end{array}$ \\
\hline SEMIA-116 & Controle & $270 \mathrm{abc}$ & $17,99 \mathrm{abc}$ \\
\hline SEMIA-116 & Thiram s & $558 \mathrm{a}$ & $31,47 \mathrm{a}$ \\
\hline SEMIA-116 & Iprodione s & $222 \mathrm{abc}$ & $19,79 \mathrm{abc}$ \\
\hline SEMIA-116 & Iprodione ss & $472 \mathrm{ab}$ & $25,72 \mathrm{ab}$ \\
\hline SEMIA-134 & Controle & $202 b c$ & $17,00 \mathrm{abc}$ \\
\hline SEMIA-134 & Thiram s & $246 \mathrm{abc}$ & $17,12 \mathrm{abc}$ \\
\hline SEMIA-134 & Iprodione s & $330 \mathrm{abc}$ & $22,20 \mathrm{abc}$ \\
\hline SEMIA-134 & Iprodione ss & $364 \mathrm{ab}$ & $25,69 \mathrm{ab}$ \\
\hline SEMIA-135 & Controle & $220 \mathrm{abc}$ & $19,47 \mathrm{abc}$ \\
\hline SEMIA-135 & Thiram s & $186 \mathrm{bc}$ & $14,85 \mathrm{bc}$ \\
\hline SEMIA-135 & Iprodione s & $198 \mathrm{bc}$ & $16,85 \mathrm{abc}$ \\
\hline SEMIA-135 & Iprodione ss & $60 c$ & $8,70 \mathrm{c}$ \\
\hline Média geral & & 277,33 & 19,74 \\
\hline CV $(\%)$ & & 30 & 34 \\
\hline
\end{tabular}

${ }^{1}$ Médias seguidas pela mesma letra não diferem entre si a $5 \%$ de probabilidade (Tukey e REGWQ).

${ }^{2}$ s: fungicida aplicado no tratamento das sementes; ss: fungicida aplicado no tratamento das sementes e na superfície do solo.

${ }^{3}$ Teste de REGWQ para diferenciação das médias (SAS, 1989).

${ }^{4}$ Teste de Tukey para diferenciação das médias (SAS, 1989). 
TABELA 4. Comparações múltiplas quanto ao efeito principal das estirpes comerciais de Rhizobium meliloti sobre as variáveis porcentagem de nitrogênio e nitrogênio total/parcela ${ }^{1}$.

\begin{tabular}{lcc}
\hline Tratamento & Nitrogênio $(\%)^{2}$ & Nitrogênio total $(\mathrm{mg})^{3}$ \\
\hline SEMIA-116 & $2,76 \mathrm{a}$ & $10,53 \mathrm{a}$ \\
SEMIA-134 & $2,60 \mathrm{ab}$ & $7,42 \mathrm{~b}$ \\
SEMIA-135 & $2,37 \mathrm{~b}$ & $3,94 \mathrm{~b}$ \\
\hline Média geral & 2,57 & 7,29 \\
\hline${ }^{1}$ Médias seguidas pela mesma letra não diferem entre si a 5\% de probabi- \\
lidade. \\
${ }^{2}$ Teste de REGWQ para diferenciação das médias (SAS, 1989). \\
${ }^{3}$ Teste de Tukey para diferenciação das médias (SAS, 1989).
\end{tabular}

SEMIA-135, e a SEMIA-134 apresentou um resultado intermediário entre elas. Os resultados obtidos neste trabalho confirmam as conclusões de Odeyemi \& Alexander (1977) e Monteiro et al. (1990), que observaram interação entre estirpes de Rhizobium e utilização de fungicidas. Måtensson (1992) não encontrou essa interação, quando trabalhou com isolados diferentes de $R$. meliloti provenientes de solos com ou sem manejo químico. De modo geral, neste trabalho, a SEMIA-116 apresentou os melhores resultados, o que pode ser evidenciado pela quantidade total de $\mathrm{N}$ colocada no sistema, uma vez que essa só dependeu da fixação biológica de $\mathrm{N}$, pois trabalhou-se com solução nutritiva sem adição de N. A quantidade de N colocada no sistema pela SEMIA-116 foi $267,25 \%$ maior que a colocada pela SEMIA-135.

\section{CONCLUSÕES}

1. As três estirpes de rizóbio apresentam o mesmo comportamento quanto à capacidade de nodulação na ausência de fungicidas; este comportamento é diferente na presença de fungicidas, ou seja, há interação entre as estirpes e os fungicidas empregados.

2. Quando se empregam os fungicidas Thiram e Iprodione, a estirpe SEMIA-116 apresenta superioridade, representada pela maior produção de matéria seca, número de nódulos, e $\mathrm{N}$ total acumulado nas plantas.
3. Não se recomenda a inoculação da estirpe de R. meliloti SEMIA-135 quando for realizada a aplicação do fungicida Iprodione na semente de alfafa, associada à aplicação na superfície do solo.

\section{REFERÊNCIAS}

ARRESE-IGOR, C.; ESTAVILLO, J.M.; PEÑA, J.I.; GONZALEZ-MURUA, C.; APARICIO-TEJO, P.M. Effect of low nitrate supply on nitrogen fixation in alfalfa root nodules induced by Rhizobium meliloti strains with varied nitrate reductase activity. Journal of Plant Physiology, v.135, p.207-211, 1989.

COMPÊNDIO de defensivos agrícolas: guia prático de produtos fitossanitários para uso agrícola. 4.ed. rev. atual. São Paulo: Andrei, 1993. 448p.

DE-POLLI, H.; SOUTO, S.M.; FRANCO, A.A. Compatibilidade de agrotóxicos com Rhizobium spp. e a simbiose das leguminosas. Seropédica: Embrapa-UAPNPBS, 1986. 75p. (EmbrapaUAPNPBS. Documentos, 3).

ISOI, T.; YOSHIDA, S. Effect of Thiram (TetramethylThiuram-Disulphide) application on nodulation in soybean and kidney bean plants: observation using the root-box-culture technique. Soil Science and Plant Nutrition, Tokyo, v.34, n.4, p.633-637, 1988.

KEPLIN, L.A.S.; SANTOS, I.R. dos. Princípios e práticas para o estabelecimento e manejo da cultura da alfafa. Suplemento Jornal da Dirat, Carambeí, v.84, p.17-23, 1991

KOLLING, J.; SCHOLLES, D.; SELBACH, P.A. Seleção de estirpes de Rhizobium para trevo subterrâneo, alfafa e cornichão. Agronomia Sulriograndense, Porto Alegre, v.19, n.2, p.103-111, 1983.

MÅTENSSON, A.M. Effects of agrochemicals and heavy metals on fast-growing Rhizobia and their symbiosis with small-seeded legumes. Soil Biology and Biochemistry, Oxford, v.24, n.5, p.435-445, 1992.

MONTEIRO, R.T.R.; BARAIBAR, A.; TSAI, S.M. Sobrevivência de Rhizobium leguminosarum bv. phaseoli em sementes tratadas com fungicidas. Revista de Microbiologia, São Paulo, v.21, n.1, p.55-59, 1990. 
ODEYEMI, O.; ALEXANDER, M. Use of fungicideresistant Rhizobia for legume inoculation. Soil Biology and Biochemistry, Oxford, v.9, p.247-251, 1977

RENNIE, R.J.; HOWARD, R.J.; SWANSON, T.A.; FLORES, G.H.A. The effect of seed-applied pesticides on growth and $\mathrm{N}_{2}$ fixation in pea, lentil and faba bean. Canadian Journal of Plant Science, Ottawa, v. 65, p.230-238, Jan. 1985.

SARRUGE, J.R. Soluções nutritivas. Summa Phytopathologica, Piracicaba, v.1, n.3, p.231-234, 1975.

SAS INSTITUTE. SAS/STAT user's guide. Version 6 , 4.ed. Cary, NC, 1989. v.2, 846p.
TU, C.M. Effect of fungicidal seed treatments on alfalfa growth and nodulation by Rhizobium meliloti. Chemosphere, London, v.10, p.127-134, 1981.

TWARY, S.N.; HEICHEL, G.H. Carbon costs of dinitrogen fixation associated with dry matter accumulation in alfalfa. Crop Science, Madison, v.31, p.985-992, July/Aug. 1991.

VANCE, C.P.; HEICHEL, G.H.; PHILLIPS, D.A Nodulation and symbiotic dinitrogen fixation. In: HANSON, A.A.; BARNES, O.K.; HILL JUNIOR, R.R. (Eds.). Alfalfa and alfalfa improvement. Madison: American Society of Agronomy, 1988. p.303-332. 